\title{
High infiltration of tumor-associated macrophages in triple-negative breast cancer is associated with a higher risk of distant metastasis
}

This article was published in the following Dove Press journal:

OncoTargets and Therapy

21 August 2014

Number of times this article has been viewed

\author{
Zhong-Yu Yuan ${ }^{1-3 *}$ \\ Rong-Zhen Luo ${ }^{1,2,4, *}$ \\ Rou-Jun Peng ${ }^{1-3}$ \\ Shu-Sen Wang ${ }^{1-3}$ \\ Cong Xue ${ }^{1-3}$
}

'State Key Laboratory of Oncology in South China, ${ }^{2}$ Collaborative Innovation Center for Cancer

Medicine, ${ }^{3}$ Departments of Medical Oncology, Sun Yat-Sen University

Cancer Center, ${ }^{4}$ Departments of Pathology, Sun Yat-Sen University Cancer Center, Guangzhou, People's Republic of China

*These authors contributed equally to this work
Correspondence: Zhong-Yu Yuan Department of Medical Oncology, Sun Yat-Sen University Cancer Center, 65I Dongfeng Road East, Guangzhou, People's Republic of China, 510060 Tel +86208734 3794

Fax +86 2087343535

Email yuanzhygz@163.com
Background: Triple-negative breast cancer (TNBC) is associated with poor prognosis and high probability of distant metastases. Tumor microenvironments play a pivotal role in tumor metastasis. Tumor-associated macrophages (TAMs) are one of the main cell components, and they are correlated with increasing metastatic risk. The aim of this study is to analyze the prognostic significance of the infiltration of TAMs in patients with TNBC.

Materials and methods: Immunohistochemical staining for cluster of differentiation (CD)68 (a marker for macrophages) was performed on tissue microarrays of operable breast cancer among 287 patients with TNBC, and the number of infiltrating TAMs was correlated with clinicopathological parameters.

Results: We found that TNBC with a large number of infiltrating TAMs had a significantly higher risk of distant metastasis, as well as lower rates of disease-free survival and overall survival than those with a smaller number of infiltrating TAMs. Multivariate analysis indicated that the number of infiltrating TAMs was a significant independent prognostic factor of disease-free survival $(P=0.001)$ in all patients.

Conclusion: Our results suggested that high infiltrating TAMs are a significantly unfavorable prognostic factor for patients with TNBC, and they could become a potentially useful prognostic marker for TNBC.

Keywords: breast carcinoma, triple-negative, tumor-associated macrophages, prognosis

\section{Introduction}

Breast cancers have been classified into distinct molecular subtypes based on deoxyribonucleic acid microarray expression profiling, which include luminal A, luminal B, human epidermal growth factor receptor-2 (HER2) overexpression, basal-like, and normal breast subtypes. ${ }^{1-3}$ These subtypes demonstrate distinctive immunohistochemical features and clinical behavior. ${ }^{4-6}$ The basal-like subtype is immunohistochemically characterized by a lack of expression of the estrogen receptor, progesterone receptor, and HER2, and it is thus also categorized as triple-negative breast cancer (TNBC). TNBC, which accounts for about $15 \%-20 \%$ of all breast cancers, is associated with poor prognosis and a high probability of distant metastases, especially of the lung and brain. ${ }^{7-10}$ As metastatic disease is incurable, accurate prognostic factors and more efficacious treatments are needed.

Inflammatory cells have gained a renewed interest in breast cancer research due to our increased understanding of their role in tumor development. Firstly, infiltrating tumor-associated macrophages (TAMs) were initially thought to represent the host response against tumors, now evidence suggests that these cells may actually 
contribute to cancer development. ${ }^{11}$ Secondly, the recruitment of TAMs changes the microenvironment, which includes the enrichment of cytokines and growth factors that may encourage the proliferation of malignant cells. ${ }^{12}$ Thirdly, the cross-talk between infiltrating TAMs and the cancer cells is likely to have profound effects on tumor progression. ${ }^{13}$ Thus, infiltrating TAMs likely promote, rather than inhibit, cancer development and progression. A large number of studies also suggested that TAMs were closely associated with poor prognosis in patients with breast cancer. ${ }^{14-17}$ However, the relationship between infiltrating TAMs and the prognosis of patients with TNBC has not yet been investigated.

In this study, we investigated the relationship of infiltrating TAMs with the clinicopathologic characteristics of TNBC.

\section{Materials and methods}

\section{Patients and tissue microarrays}

This study consisted of 287 female patients with TNBC diagnosed without any evidence of distant metastasis at the time of surgery, between January 2000-December 2008 at Sun Yat-sen University Cancer Center (Guangdong, People's Republic of China). The cases selected were based on the distinctive pathologic diagnosis of invasive breast cancer, the availability of complete medical records and patients' follow-up status, and the lack of estrogen receptor, progesterone receptor, and HER2 expression. All patients were staged according to the American Joint Committee on Cancer (AJCC 2010, seventh edition) tumor, node, metastasis (TNM) Staging System for Breast Cancer. ${ }^{18}$

In this study, the paraffin-embedded pathologic specimens were retrieved from the archives of the Department of Pathology of Sun Yat-sen University Cancer Center. Tissue microarrays were constructed as described previously. ${ }^{19}$ Each sample was arrayed in triplicate to minimize tissue loss and to overcome tumor heterogeneity. All sample donors gave written informed consent. The use of the samples involved in our study was approved by the Institute Research Medical Ethics Committee of Sun Yat-sen University.

\section{Immunohistochemistry}

Immunohistochemistry was performed for cluster of differentiation (CD)68 on tissue microarray sections. Briefly, tissue microarray slides were deparaffinized and rehydrated for 5 minutes. After microwave pretreatment in citrate buffer ( $\mathrm{pH}$ 6.0) for antigen retrieval, the slides were immersed in $0.3 \%$ (volume/volume) hydrogen peroxide for 20 minutes to block endogenous peroxidase activity. The slides were then washed and incubated overnight at $4^{\circ} \mathrm{C}$ with primary antibodies against CD68 (RM-9116, rabbit, 1:100; Lab Vision, Thermo Fisher Scientific, Waltham, MA, USA). After a second incubation with biotinylated antigoat antibodies, the slides were incubated with peroxidase-labeled streptavidin. The reaction products were visualized by immersing the slides in diaminobenzidine tetrachloride and counterstaining with Harris hematoxylin.

\section{Evaluation of immunohistochemistry}

TMAs were analyzed by one of the authors (RZL). The evaluation of full-face tissue sections revealed that macrophages were observed in both tumor nest and stroma. Recently, several studies have showed that TAMs in tumor stroma, but not in tumor nest, were connected with a worse survival rate for patients with breast cancer. ${ }^{20}$ We thus counted the number of TAMs in three separate tumor stroma sites. The number of positive CD68 cells (cytoplasmic staining for macrophages) in the stroma was counted in three high-power fields $(200 \times$ objective) per TMA tissue core, and then the mean number of triplicate cores from each tumor was calculated for the macrophages.

\section{Statistical analysis}

The $\mathrm{R}$ program maxstat (Institute for Statistics and Mathematics, Wirtschaftsuniversität Wien, Vienna, Austria) was used to find an optimal cut-off point for macrophages as a predictor of cancer recurrence. We found that sixteen macrophages per high-power field was the optimal cutoff point that provided a good separation of the time-to-recurrence curves. Then, the patients were divided into one of two groups: the TAM high-infiltration group ( $\geq 16$ TAMs); and the TAM low-infiltration group $(<16$ TAMs). We examined the relationship between macrophages and the clinicopathologic variables by the $\chi^{2}$ test or Fisher's exact test. The disease-free survival (DFS) rate and overall survival (OS) rate curves were estimated using the Kaplan-Meier method, and the survival differences according to CD68 expression were compared using the log-rank test. A multivariate analysis was performed using Cox's regression model. To examine whether macrophages were an independent prognostic factor, we adjusted for age at diagnosis, tumor size, lymph node status, histological grade, and lymphovascular invasion (LVI), which were considered as traditional prognostic factors, were included in the multivariate analysis with Enter model. The hazard ratios were presented with their $95 \%$ confidence intervals. 
$P$-values $\leq 0.05$ denote statistical significance. All analyses were performed using the statistical software, SPSS 16.0 (IBM Corporation, Armonk, NY, USA).

In addition, DFS was defined as the interval from the date of operation to the date of the first recurrence. OS was calculated as the period from the date of diagnosis to the date of death. Locoregional relapse-free survival was defined as the interval from the first treatment for breast cancer to the first locoregional relapse. Distant metastasis free survival was calculated as the period from the date of diagnosis to the date of distant metastasis or the presence of contralateral breast cancer. Locoregional relapse was defined as the recurrence either in the treated breast or in the ipsilateral lymph node-bearing area (axillary, internal mammary, supraclavicular node).

\section{Results}

\section{Association of TAMs with clinicopathologic characteristics}

A total of 287 TNBC patients were grouped according to the mean number of TAMs. Among the 287 tumor specimens, 103 (35.9\%) were divided into the high-infiltration group (Figure 1). The association of TAMs with the various clinicopathological parameters is listed in Table 1. TAMs were associated with lymph node involvement $(P=0.007)$ and LVI $(P=0.032)$. There was no significant association with age at diagnosis, tumor size, and histological grade between the two groups.

Table I Association of TAMs with various clinicopathological features

\begin{tabular}{|c|c|c|c|c|c|}
\hline \multirow[t]{2}{*}{ Characteristics } & \multicolumn{2}{|c|}{ High TAMs } & \multicolumn{2}{|c|}{ Low TAMs } & \multirow[t]{2}{*}{$P$-value } \\
\hline & $\mathbf{N}$ & $\%$ & $\mathbf{N}$ & $\%$ & \\
\hline$N$ & 103 & 35.9 & 184 & 64.1 & - \\
\hline \multicolumn{6}{|l|}{ Age, years } \\
\hline$\leq 40$ & 27 & 26.2 & 40 & 21.7 & 0.388 \\
\hline$>40$ & 76 & 73.8 & 144 & 78.3 & \\
\hline \multicolumn{6}{|l|}{ Tumor size, cm } \\
\hline$\leq 2$ & 26 & 25.2 & 41 & 22.3 & 0.565 \\
\hline$>2$ & 77 & 74.8 & 143 & 77.7 & \\
\hline \multicolumn{6}{|l|}{ Node status } \\
\hline Negative & $4 I$ & 39.8 & 105 & 57.1 & $0.007^{*}$ \\
\hline Positive & 62 & 60.2 & 79 & 42.9 & \\
\hline \multicolumn{6}{|l|}{ Histological grade } \\
\hline $1 / 2$ & 23 & 22.3 & 60 & 32.6 & 0.078 \\
\hline 3 & 80 & 77.7 & 124 & 67.4 & \\
\hline \multicolumn{6}{|c|}{ Lymphovascular invasion } \\
\hline Absent & 63 & 61.2 & 136 & 73.9 & $0.032 *$ \\
\hline Present & 40 & 38.8 & 48 & 26.1 & \\
\hline
\end{tabular}

Note: *Statistically significant.

Abbreviations: TAM, tumor-associated macrophage; $\mathrm{N}$, number.

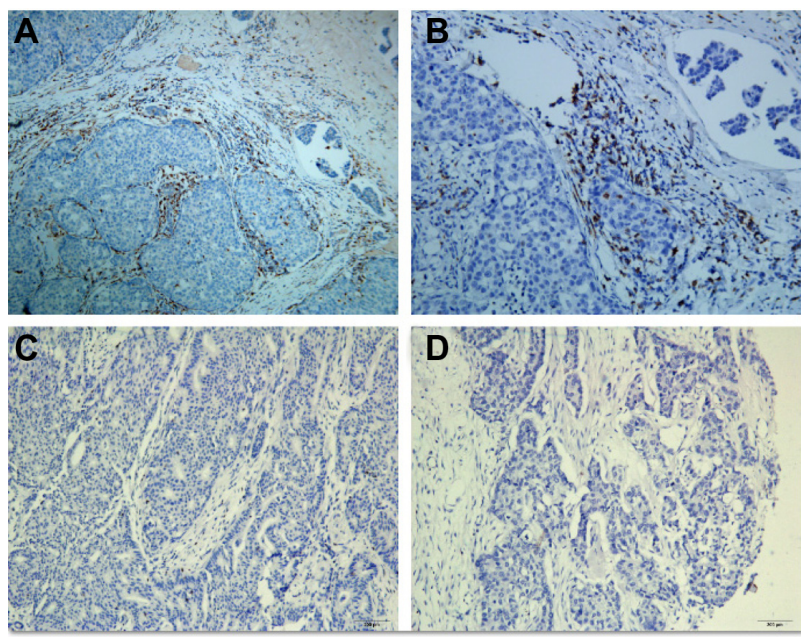

Figure I Representative immunohistochemical staining results for tumor-associated macrophages (marker CD68).

Notes: (A) High infiltration (100x); (B) high infiltration (200x); (C) low infiltration (100x); (D) low infiltration (200x).

Abbreviation: CD68, cluster of differentiation 68 .

\section{TAMs and disease-free survival and overall survival}

As of December 2013, the median follow-up time was 89 months (range: 4-181 months). Among the total of 287 patients, 106 relapsed and 72 died. The 5-year DFS and OS of all patients were $79.0 \%$ and $83.1 \%$, respectively. When the patients were stratified in terms of infiltrating TAMs, the 8-year DFS for the TAM high-infiltration and TAM low-infiltration patients were $67.8 \%$ and $85.3 \%$, respectively. The 5-year OS for the TAM high-infiltration and TAM low-infiltration patients were $71.1 \%$ and $89.5 \%$, respectively (Figure 2A and B).

Compared to the TAM low-infiltration patients, patients with TAM high-infiltration had a significantly higher risk for developing distant metastasis $(72.1 \%$ versus $86.8 \%$; $P<0.001$ ), whereas the risk for locoregional recurrence was not significantly different between these two groups (82.7\% versus $85.1 \%$, respectively; $P=0.123$ ) (Figure $2 \mathrm{C}$ and $\mathrm{D}$ ).

\section{Univariate and multivariate analysis}

Statistically significant predictors of DFS, as observed during the univariate analysis, are listed in Table 2. Large primary tumor, positive lymph nodes, higher histological grade, present LVI, and high TAM infiltration were correlated with shorter DFS. The patients with high TAM infiltration had significantly worse outcomes in terms of DFS $(P<0.001)$ than did the patients with low TAM infiltration. In the multivariate analysis, high TAM infiltration remained a significant predictor of DFS when entered into a model containing all of the clinicopathologic variables $(P<0.001)$. 
A

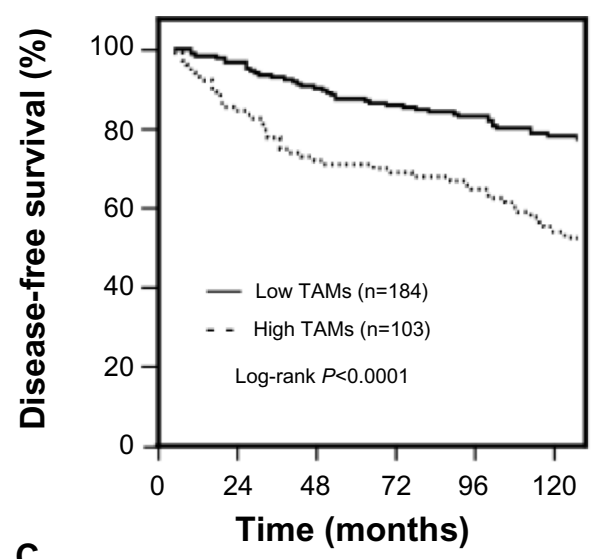

C

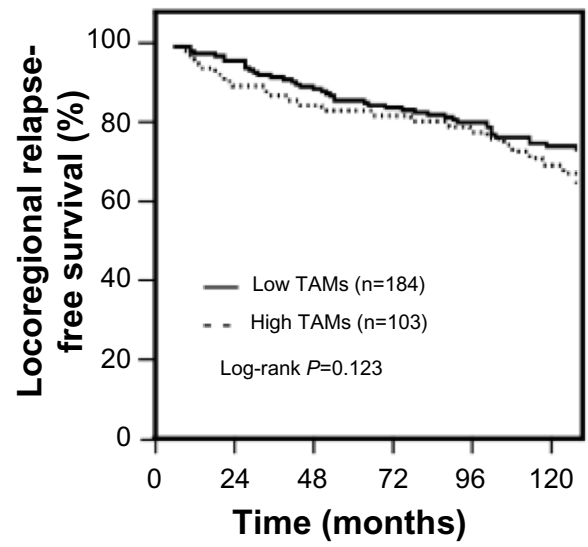

B

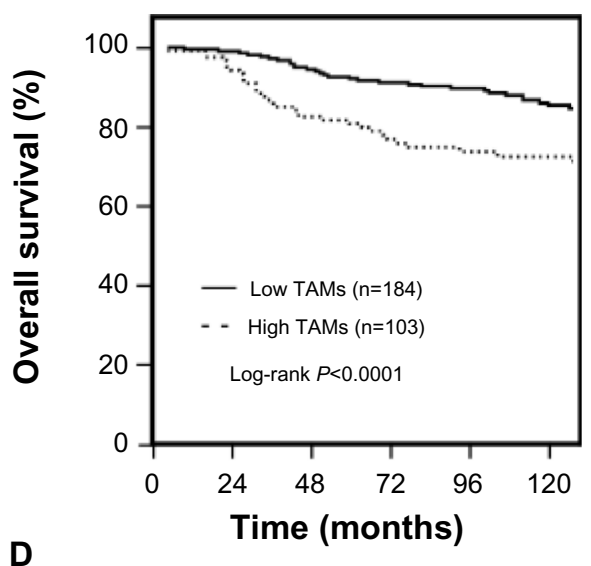

D

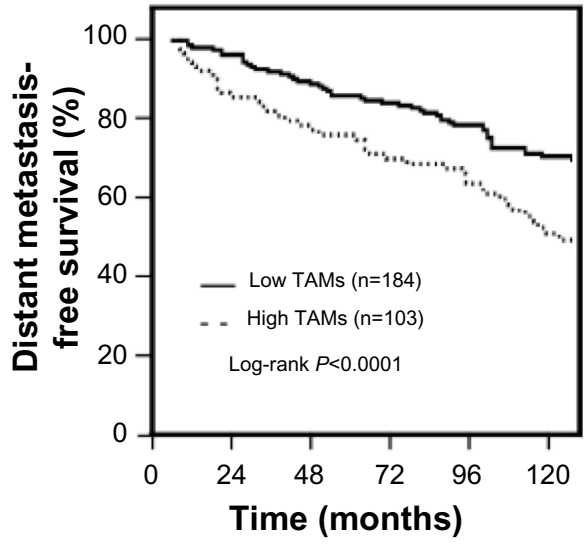

Figure 2 Association of TAM infiltration with the prognosis of TNBC patients.

Notes: (A) Disease-free survival; (B) overall survival; (C) locoregional relapse-free survival; (D) distant metastasis-free survival.

Abbreviations: TAM, tumor-associated macrophage; n, number; TNBC, triple-negative breast cancer.

\section{Discussion}

In the current study, we evaluated the prognostic significance of TAMs in patients with TNBC. We found that patients with high TAM infiltration accounted for $35.9 \%$ of 287 TNBC cases. High TAM infiltration was associated with a higher proportion of positive lymph nodes and LVI. Patients with high TAM infiltration had a significantly higher risk for developing distant metastasis and poor OS and DFS. Multivariate analysis showed that TAM infiltration was an independent predictor of poor DFS among TNBC patients.
Over the past few years, a growing amount of evidence has shown that the tumor microenvironment, which is mainly composed of leukocytes, vascular cells, and fibroblasts, plays a pivotal role in tumor progression and metastasis. ${ }^{21,22} \mathrm{In}$ the tumor microenvironment, infiltrating leukocytes were recently suggested to play a key role in breast cancer. ${ }^{23}$ TAMs make up the majority of infiltrating leukocytes. It has been suggested that high TAM infiltration is correlated with increasing metastatic risk and poor prognosis in breast cancer. ${ }^{24-27}$ A meta-analysis showed that in $>80 \%$ of cases,

Table 2 Univariate and multivariate analysis of disease-free survival in the entire study population

\begin{tabular}{|c|c|c|c|c|c|c|}
\hline \multirow[t]{2}{*}{ Variables } & \multicolumn{3}{|c|}{ Univariate } & \multicolumn{3}{|c|}{ Multivariate } \\
\hline & HR & $95 \% \mathrm{Cl}$ & $P$-value & HR & $95 \% \mathrm{Cl}$ & $P$-value \\
\hline Age, years $(\leq 40$ versus $>40)$ & 0.88 & $0.59-1.31$ & 0.527 & 0.92 & $0.6 \mathrm{I}-\mathrm{I} .38$ & 0.685 \\
\hline Tumor size, $\mathrm{cm}(\leq 2$ versus $>2)$ & 1.75 & $1.06-2.89$ & 0.019 & 1.61 & $0.97-2.68$ & 0.067 \\
\hline Node status (negative versus positive) & 4.13 & $2.76-6.18$ & $<0.001$ & 2.33 & $1.44-3.79$ & 0.001 \\
\hline Histological grade ( $1 / 2$ versus 3$)$ & 2.36 & $1.46-3.82$ & $<0.001$ & 1.45 & $0.89-2.38$ & 0.140 \\
\hline Lymphovascular invasion (absent versus present) & 3.75 & $2.62-5.35$ & $<0.001$ & 1.99 & $1.3 \mathrm{I}-3.03$ & 0.001 \\
\hline TAM infiltration (low versus high) & 2.77 & $1.94-3.96$ & $<0.001$ & 2.20 & $1.53-3.16$ & $<0.001$ \\
\hline
\end{tabular}

Abbreviations: $\mathrm{HR}$, hazard ratio; $\mathrm{Cl}$, confidence interval; TAM, tumor-associated macrophage. 
increased macrophage density was associated with poor prognosis. ${ }^{28}$ However, it was particularly noteworthy that the infiltration of macrophages was mainly associated with hormone receptor negativity and basal phenotype. ${ }^{20,29}$ These results suggested that TAM infiltration might be more closely associated with TNBC.

The mechanism underlying TAM's ability to influence cancer progression and metastasis remains unclear. In the tumor microenvironment, macrophages are derived from circulating monocytes. ${ }^{30}$ Several cytokines have been implicated in the recruitment of macrophages into the tumors. ${ }^{28}$ In turn, TAMs produce a variety of cytokines and chemokines, as well as growth factors for both the epithelial and endothelial cells, which play a vital role in tumor growth and metastasis. ${ }^{12,31}$

\section{Conclusion}

TAM infiltration might serve as a prognostic indicator for poor outcomes among patients with TNBC. Perhaps more importantly, these cells may serve as potential cellular targets for novel therapeutic interventions.

\section{Acknowledgments}

This study was financially supported by the Science and Technology Program of Guangdong Province (No 2010B031600219) and by the National Natural Science Foundation of China (Grant No 81372820). The funders had no role in the study design, data collection and analysis, the decision to publish, or in the preparation of the manuscript.

\section{Disclosure}

The authors report no conflicts of interest in this work.

\section{References}

1. Perou CM, Sørlie T, Eisen MB, et al. Molecular portraits of human breast tumours. Nature. 2000;406(6797):747-752.

2. Sørlie T, Perou CM, Tibshirani R, et al. Gene expression patterns of breast carcinomas distinguish tumor subclasses with clinical implications. Proc Natl Acad Sci U S A. 2001;98(19):10869-10874.

3. Sorlie T, Tibshirani R, Parker J, et al. Repeated observation of breast tumor subtypes in independent gene expression data sets. Proc Natl Acad Sci U S A. 2003;100(14):8418-8423.

4. Carey LA, Perou CM, Livasy CA, et al. Race, breast cancer subtypes, and survival in the Carolina Breast Cancer Study. JAMA. 2006;295(21): 2492-2502.

5. Desmedt C, Haibe-Kains B, Wirapati P, et al. Biological processes associated with breast cancer clinical outcome depend on the molecular subtypes. Clin Cancer Res. 2008;14(16):5158-5165.

6. Nielsen TO, Hsu FD, Jensen K, et al. Immunohistochemical and clinical characterization of the basal-like subtype of invasive breast carcinoma. Clin Cancer Res. 2004;10(16):5367-5374.

7. Dent R, Trudeau M, Pritchard KI, et al. Triple-negative breast cancer: clinical features and patterns of recurrence. Clin Cancer Res. 2007; 13(15 Pt 1):4429-4434.
8. Tischkowitz M, Brunet JS, Bégin LR, et al. Use of immunohistochemical markers can refine prognosis in triple negative breast cancer. $B M C$ Cancer. 2007;7:134.

9. Bauer KR, Brown M, Cress RD, Parise CA, Caggiano V. Descriptive analysis of estrogen receptor (ER)-negative, progesterone receptor (PR)-negative, and HER2-negative invasive breast cancer, the so-called triple-negative phenotype: a population-based study from the California cancer Registry. Cancer. 2007;109(9):1721-1728.

10. Foulkes WD, Smith IE, Reis-Filho JS. Triple-negative breast cancer. N Engl J Med. 2010;363(20):1938-1948.

11. Pollard JW. Macrophages define the invasive microenvironment in breast cancer. J Leukoc Biol. 2008;84(3):623-630.

12. Coussens LM, Werb Z. Inflammation and cancer. Nature. 2002;420(6917):860-867.

13. Mahmoud SM, Lee AH, Paish EC, Macmillan RD, Ellis IO, Green AR. The prognostic significance of B lymphocytes in invasive carcinoma of the breast. Breast Cancer Res Treat. 2012;132(2):545-553.

14. Bolat F, Kayaselcuk F, Nursal TZ, Yagmurdur MC, Bal N, Demirhan B. Microvessel density, VEGF expression, and tumor-associated macrophages in breast tumors: correlations with prognostic parameters. J Exp Clin Cancer Res. 2006;25(3):365-372.

15. Goswami S, Sahai E, Wyckoff JB, et al. Macrophages promote the invasion of breast carcinoma cells via a colony-stimulating factor-1/ epidermal growth factor paracrine loop. Cancer Res. 2005;65(12): 5278-5283.

16. Ueno T, Toi M, Saji H, et al. Significance of macrophage chemoattractant protein-1 in macrophage recruitment, angiogenesis, and survival in human breast cancer. Clin Cancer Res. 2000;6(8): 3282-3289.

17. Wyckoff J, Wang W, Lin EY, et al. A paracrine loop between tumor cells and macrophages is required for tumor cell migration in mammary tumors. Cancer Res. 2004;64(19):7022-7029.

18. Edge SB, Byrd DR, Compton CC. AJCC Cancer Staging Manual (ed 7). Chicago, IL. Springer, 2009.

19. He J, Peng R, Yuan Z, et al. Prognostic value of androgen receptor expression in operable triple-negative breast cancer: a retrospective analysis based on a tissue microarray. Med Oncol. 2012;29(2): 406-410.

20. Medrek C, Pontén F, Jirström K, Leandersson K. The presence of tumor associated macrophages in tumor stroma as a prognostic marker for breast cancer patients. BMC Cancer. 2012;12:306.

21. Robinson BD, Sica GL, Liu YF, et al. Tumor microenvironment of metastasis in human breast carcinoma: a potential prognostic marker linked to hematogenous dissemination. Clin Cancer Res. 2009;15(7):2433-2441.

22. Bhowmick NA, Neilson EG, Moses HL. Stromal fibroblasts in cancer initiation and progression. Nature. 2004;432(7015):332-337.

23. Ben-Baruch A. Host microenvironment in breast cancer development: inflammatory cells, cytokines and chemokines in breast cancer progression: reciprocal tumor-microenvironment interactions. Breast Cancer Res. 2003;5:31-36.

24. Paik S, Shak S, Tang G, et al. A multigene assay to predict recurrence of tamoxifen-treated, node-negative breast cancer. $N$ Engl J Med. 2004;351(27):2817-2826.

25. Mukhtar RA, Nseyo O, Campbell MJ, Esserman LJ. Tumor-associated macrophages in breast cancer as potential biomarkers for new treatments and diagnostics. Expert Rev Mol Diagn. 2011;11(1):91-100.

26. DeNardo DG, Brennan DJ, Rexhepaj E, et al. Leukocyte complexity predicts breast cancer survival and functionally regulates response to chemotherapy. Cancer Discov. 2011;1(1):54-67.

27. Leek RD, Lewis CE, Whitehouse R, Greenall M, Clarke J, Harris AL. Association of macrophage infiltration with angiogenesis and prognosis in invasive breast carcinoma. Cancer Res. 1996;56(20): 4625-4629.

28. Bingle L, Brown NJ, Lewis CE. The role of tumour-associated macrophages in tumour progression: implications for new anticancer therapies. J Pathol. 2002;196(3):254-265.

29. Mahmoud SM, Lee AH, Paish EC, Macmillan RD, Ellis IO, Green AR. Tumour-infiltrating macrophages and clinical outcome in breast cancer. J Clin Pathol. 2012;65(2):159-163. 
30. Vicioso L, Gonzalez FJ, Alvarez M, et al. Elevated serum levels of vascular endothelial growth factor are associated with tumor-associated macrophages in primary breast cancer. Am J Clin Pathol. 2006;125(1): $111-118$.
31. Lewis CE, Pollard JW. Distinct role of macrophages in different tumor microenvironments. Cancer Res. 2006;66(2):605-612.

\section{Publish your work in this journal}

OncoTargets and Therapy is an international, peer-reviewed, open access journal focusing on the pathological basis of all cancers, potential targets for therapy and treatment protocols employed to improve the management of cancer patients. The journal also focuses on the impact of management programs and new therapeutic agents and protocols on patient perspectives such as quality of life, adherence and satisfaction. The manuscript management system is completely online and includes a very quick and fair peer-review system, which is all easy to use. Visit http://www.dovepress.com/testimonials.php to read real quotes from published authors.

\footnotetext{
Submit your manuscript here: http://www.dovepress.com/oncotargets-and-therapy-journal
} 